\title{
Simulation of non-contact tonometer-Ocular response analyzer
}

\author{
Mohammad Arsalan Khan \\ Division of Civil Engineering, University of Dundee, Dundee, DD1 4HN, Scotland, UK \\ Department of Civil Engineering, Zakir Husain College of Engineering \& Technology, Aligarh Muslim University, U.P., India
}

Received: 12 March 2016, Revised: 12 March 2016, Accepted: 30 March 2016

Published online: 10 April 2016

\begin{abstract}
As per World Health Organization, Glaucoma is considered as the second leading cause of irreversible blindness worldwide. An accurate assessment of Intraocular Pressure (IOP) is crucial for diagnosis and management of a chronic eye disease called Glaucoma. The elevation of IOP in eye leads to optic nerve damage and hence causing visual impairment. Thus, IOP measurement in tonometry has become an essential part of routine eye examinations for the diagnosis, screening and managing response to treatment in patients.

Simultaneous explosion of ophthalmic knowledge and medical instrument, being made in the 19th century, has led to the invention of tonometers of varied designs and principles, and Non-Contact Tonometers (NCTs) are among them. Glodmann Applanation Tonometer (GAT) is considered the 'gold standard' in measuring IOP; however, IOP measurement using GAT is now known to be affected by various factors like corneal thickness, curvature and material properties as demonstrated by Khan [1]. Due to inaccuracies in measuring IOP by GAT, this 'gold standard' has been challenged. Therefore, the present research aims to develop a multi-parametric correction equation to determine the True Intraocular Pressure (IOPT) using Non-Contact Tonometer and the current article focuses on evaluating the influence of individual parameters on IOP by NCT.
\end{abstract}

Keywords: Cornea, intraocular pressure, tonometry, glaucoma, numerical modeling.

\section{Introduction}

Glaucoma is a chronic eye disease caused by elevation in the Intraocular Pressure (IOP) of human eye and is stated to be the second leading cause of irreversible blindness worldwide, and around 66.8 million people are suspected to be victimised by the disease worldwide in the year 2000 [2]. Moreover, it is expected that the number of people affected by Glaucoma will increase considerably with the world's aging population. The fact that this disease doesn't show any major symptoms during its early development stages explains its criticality. The elevation of the IOP leads to optic nerve damage ultimately leading to visual impairment. Thus, IOP measurement has become an essential part of routine eye examinations for the diagnosis, screening and managing response to treatment in patients suffering from the said disease. Tonometer is a device which is used to gauge the IOP of the eye. The Goldmann Applanation Tonometer (GAT) is considered as the 'gold standard' for measuring IOP of the eye [3]. However, IOP measurement by GAT is now known to be affected by variations in central corneal thickness (CCT), curvature (R) and material properties of eye [4, 5, 6]. Due to such inaccuracies, its 'gold standard' has been challenged. While research is progressing to establish correction factors to improve IOP measurement by GAT [1], new tonometers of varying designs and principles have been developed with the aim of determining IOP estimates free from the variations in CCT, R and material properties; Non-Contact Tonometers (NCTs) are among them. The NCTs work on applanation principle similar to GAT. Different types of NCTs are available in the market popular among them are Ocular Response Analyzer (ORA: Reichert Corporation; Philadelphia, PA), Keeler PULSAIR (Keeler Ltd, Windsor, U.K), Reichert AT550 (Depew, NY, USA), Topcon CT60 (Topcon Corporation, 
Tokyo, Japan), Nidek NT4000 (Nidek Co. Ltd., Aichi, Japan) and Canon-TX10 (Canon USA Inc, One Canon Plaza, Lake Success, NY, USA). They use an air puff to create an applanation event on the human cornea. The change in the characteristics of the corneal light reflex produced by the air puff is measured electronically in NCTs. Unlike GAT, the main advantage of a NCT is that it requires no corneal contact; thereby decreasing the risk of disease transmission. Moreover, a NCT is patient friendly as no topical anesthesia is required in recording IOP.

Practical Significance. In a study by Martinez-de-la-Casa et al. [7], 48 eyes of 48 patients suffering from Glaucoma were examined using ORA \& GAT. A mean difference of $7.2 \mathrm{~mm} \mathrm{Hg}$ between GAT IOP and IOPG and a mean difference of $8.3 \mathrm{~mm} \mathrm{Hg}$ between GAT IOP and IOGCC were recorded in the study. Thus, in both cases ORA measurement overestimated the GAT IOP. In another study by Kotecha et al. [8], 144 eyes of 144 untreated subjects were examined. In the study a factor termed 'Corneal Compensation Factor (CCF)' was introduced and the corresponding IOP was termed IOPCCF. The mean difference between GAT IOP and IOPCCF was found to be $0.1 \mathrm{~mm} \mathrm{Hg}$, while the mean difference between GAT IOP and IOPCC was $1.7 \mathrm{~mm} \mathrm{Hg}$. IOPCC on an average overestimated GAT IOP as found in the study. In another study by Lam et al. [9], 125 normal Chinese subjects were examined using ORA \& GAT. The study demonstrated a better agreement between GAT and ORA than the previous two studies. A mean difference of just 0.33 $\mathrm{mm} \mathrm{Hg}$ between IOPG and GAT IOP and $0.24 \mathrm{~mm} \mathrm{Hg}$ between IOPCC and GAT IOP was established in the study. Therefore, preference is made that it should be ensured that IOP readings are taken using modern tonometers and factors affecting the IOP readings are recognised so that correct IOP measurement can be made.

The present study aims to develop a multi-parametric correction equation for determining True Intraocular Pressure (IOPT) using NCT. The correction equation can be developed by considering the simultaneous effect of variations in $\mathrm{CCT}, \mathrm{R}$ and material properties. The study adopts a prudent methodology of predictive non-linear finite element numerical modeling to simulate the ORA procedure using ABAQUS software. Moreover, as a single equation, the correction equation is presented in a simple form suitable for clinical use.

\section{Method}

As outlined by Khan [1], many studies have been carried out in order to develop a correction equation for estimating true IOP by GAT independent of variations in corneal parameters like CCT, R and material properties. But sadly, not much of work has been done in analyzing the effect of these parameters on IOP measured by NCT. A parametric study will be conducted to gauge the effect various corneal parameters have on pressure measurements by NCT. The present study aims to improve the IOP estimation through NCT by developing a muti-paramateric correction equation which considers the simultaneous effect of variations in CCT, R and material properties; before which, the effect of individual parameters need to be accessed. The correction will be presented in a simple form suitable for clinical applications. In order to achieve this, representative non-linear finite element numerical simulation of the NCT procedure has been employed using ABAQUS software. The numerical models used in this study consider more realistic in-vivo behaviour of human eye like hyperelasicity, hysteresis and real- life cornea-sclera connection. Moreover, the findings of the parametric study are also clinically relevant as they will signify the importance of various corneal parameters and can give ophthalmologists an idea of the influence (if any) these parameters have on IOP measured by NCTs.

\subsection{Finite element modeling using Abaqus}

Numerical modeling based on non-linear finite element analysis has been used in the present study to enable a detailed representation of biomechanical behaviour of human cornea. Numerical modeling possesses the potential to represent realistic conditions without having to adopt the simplifications necessary with mathematical closed form solutions. 
Table 1: Parameter and corresponding range of values.

\begin{tabular}{|l|l|}
\hline Parameter & Range of values considered in the study \\
\hline CCT $[\mathrm{mm}]$ in steps of $100 \mu \mathrm{m}$ & $0.445,0.545,0.645$ \\
\hline Change in corneal radius $\mathrm{R}[\mathrm{mm}]$ in steps of $0.6 \mathrm{~mm}$ & $7.2,7.8,8.4$ \\
\hline Hyperelasticity related to age & $50-64$ years, 65-79 years, $80-95$ years \\
\hline Change of Intraocular Pressure (IOP) $[\mathrm{mm} \mathrm{Hg}]$ & $10,15,20$ \\
\hline
\end{tabular}

Human cornea possesses complex structure, both at the microscopic and macroscopic levels. Based on past research, the present study strikes the balance between cost and accuracy by distinguishing between the parameters that have a considerable effect and the parameters which can be ignored for their negligible effect on the corneal behaviour.

ABAQUS is the finite element package which has been employed in the present research for creating the numerical models and simulating the NCT procedure. ABAQUS VIEWER has been used to measure the deformations and reporting the behaviour of the corneal models. Applying symmetries in two planes along superior-inferior (vertical) and temporal-nasal (horizontal) directions, the FE model is shown in Fig. 1. The features like cornea's hyperselastic, hysteric and anisotropic behaviour, multi-layer construction, weak inter-laminar adhesion, non-uniform thickness, elliptical topography and connection to sclera have been optimised to improve the efficiency of the corneal models.

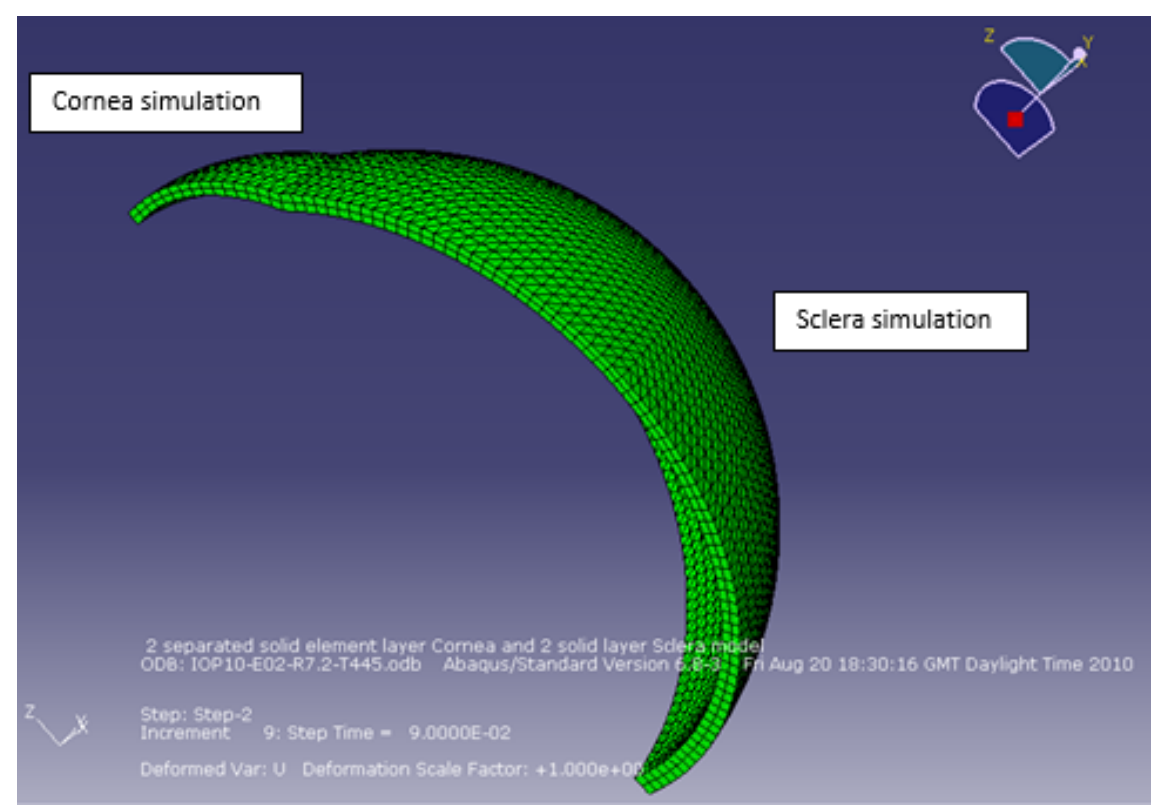

Fig. 1: FE simulation model of eye ball.

To conduct the parametric numerical study, a wide range of values for corneal thickness, curvature, age and IOP have been considered as summarised in Table 1.

Thus, in the present study altogether, 81 different Corneal Models have been generated for comparison.

Similar to Khan [1], the following stress $(\sigma)$-strain $(\varepsilon)$ relationships handles the hyperelasticity within the material model, gathered through the testing of excised cornea tested for age range of 50 to 95 years old:

$\sigma_{\text {loading }}(M P a)=0.00480\left(e^{64.8 d \varepsilon}-1\right)$, 
where,

$d=1-0.0037\left(87.5-\right.$ age $\left._{\text {years }}\right)$.

$\sigma_{\text {unloading }}(M P a)=0.00036\left(e^{355 f \varepsilon}-1\right)$,

where,

$f=1-0.0088\left(87.5-\right.$ age $\left._{\text {years }}\right)$.

\subsection{Optimization for Developing the Correction Equation}

In order to develop the multi-parametric correction equation, a Database Excel Sheet (Fig. 2) is formed containing the input values (CCT, R, Age and IOP) and the output values $\left(\mathrm{P}_{1}\right)$. With the use of Excel solver, the multi-parametric correction equation are put in the following form of single equation:

$$
K=\frac{P_{1}}{I O P T}=A_{C C T} \cdot A_{R} \cdot A_{a g e} \cdot A_{P_{1}}
$$

where,

$A_{C C T}=$ effect of variation in CCT (mm)

$A_{R}=$ effect of variation in $\mathrm{R}(\mathrm{mm})$

$A_{\text {age }}=$ effect of variation in age (years)

$A_{P_{1}}=$ effect of variation in $P_{1}$. (mm Hg)

\section{Results}

This section presents the findings of the parametric study that are conducted to analyse the effect of various corneal parameters on pressure measurements performed by NCT. In order to make an effort to accommodate the errors in tonometry readings subjected to the of variations in various corneal parameters; a multi-dimensional parametric study is conducted to assess the effects of variations in corneal thickness, curvature and age on the accuracy of first applanation pressure $\left(\mathrm{P}_{1}\right)$ measurement made by NCT within the ranges shown in Table 1.

To show the effect on material stiffness, the age range over which the numerical models are valid, that is 50-95 years, is further divided into three sub-ranges as follows-

-Young Age Range: 50-64 years with an average age $=57$ years

-Middle Age Range: $65-79$ years with an average age $=72$ years

-Old Age Range: $80-95$ years with an average age $=87.5$ years

A numerical model with specific values of CCT, R, age and IOP are created for each case.

\subsection{Effect of variation in CCT}

The numerical estimation of the effect of CCT on $\mathrm{P}_{1}$ measurement is shown in Fig. 3 for corneas with CCT ranging between $445 \mu \mathrm{m}$ and $645 \mu \mathrm{m}$; age range is young, middle and old (average age $=57,72$ and 87.5 years respectively); and IOPT values of $10 \mathrm{~mm} \mathrm{Hg}, 15 \mathrm{~mm} \mathrm{Hg}$ and $20 \mathrm{~mm} \mathrm{Hg}$. The value of $\mathrm{R}$ is kept constant at a value of $7.8 \mathrm{~mm}$ in all cases. Fig. 3 shows a slightly non-linear increase in $\mathrm{P}_{1}$ with higher CCT. This could be attributed to the fact that a close association exists between CCT and stiffness of cornea. Moreover, the relative error in pressure measurement is found to be affected by the IOPT level; for example, with CCT $=545 \mu \mathrm{m}$ and young age group (average age $=57$ years), $\mathrm{P}_{1} / \mathrm{IOPT}$ is $0.976,0.656$, and 0.497 under IOPT $=10 \mathrm{~mm} \mathrm{Hg}, 15 \mathrm{~mm} \mathrm{Hg}$ and $20 \mathrm{~mm} \mathrm{Hg}$ respectively. 


\begin{tabular}{|c|c|c|c|c|c|c|c|c|}
\hline 4 & A & B & c & D & E & $F$ & G & H \\
\hline 1 & & & \\
\hline 2 & & IOPT (mm $\mathrm{Hg})$ & Age (years) & $\mathrm{CCT}(\mathrm{mm})$ & $R(\mathrm{~mm})$ & P1 (MPa) & P1 ( $\mathrm{mm} \mathrm{Heg}$ & \\
\hline 4 & & 10 & 57 & 0.445 & 7.2 & 0.001167 & 8.7525 & \\
\hline 5 & & 10 & 57 & 0.445 & 7.8 & 0.00114 & 8.55 & \\
\hline 6 & & 10 & 57 & 0.445 & 8.4 & 0.001126 & 8.445 & \\
\hline 7 & & 10 & 57 & 0.545 & 7.2. & 0.001350 & 10.17 & \\
\hline 8 & & 10 & 57 & 0.545 & 7.8 & 0.001301 & 9.7575 & \\
\hline 9 & & 10 & 57 & 0.545 & 8.4 & 0.00127 & 9.525 & \\
\hline 10 & & 10 & 57 & 0.645 & 7.2 & 0.001612 & 12.09 & \\
\hline 11 & & 10 & 57 & 0.645 & 7.8 & 0.001514 & 11.355 & \\
\hline 12 & & 10 & 57 & 0.645 & 8.4 & 0.001458 & 10.92 & \\
\hline 13 & & 10 & 72 & 0.445 & 7.2 & 0.001228 & 9.21 & \\
\hline 14 & & 10 & $n$ & 0.445 & 7.8 & 0.001228 & 9.21 & \\
\hline 15 & & 10 & $n$ & 0.445 & 8.4 & 0.001194 & 8.955 & \\
\hline 16 & & 10 & 72 & 0.545 & 7.2 & 0.001655 & 12.6375 & \\
\hline 17 & & 10 & 72 & 0.545 & 7.8 & 0.001477 & 11.0775 & \\
\hline 18 & & 10 & 72 & 0.545 & 8.4 & 0.001376 & 10.32 & \\
\hline 19 & & 10 & 72 & 0.645 & 7.2 & 0.002308 & 17.31 & \\
\hline 20 & & 10 & 72 & 0.645 & 7.8 & 0.001894 & 14.205 & \\
\hline 21 & & 10 & $n$ & 0.645 & 8.4 & 0.001669 & 12.5175 & \\
\hline 22 & & 10 & 87.5 & 0.445 & 7.2 & 0.001442 & 20.815 & \\
\hline 23 & & 10 & 87.5 & 0.445 & 7.8 & 0.001442 & 10.815 & \\
\hline 24 & & 10 & 87.5 & 0.445 & 8.4 & 0.001353 & 10.1475 & \\
\hline 25 & & 10 & 87.5 & 0.545 & 7.2 & 0.002338 & 17.535 & \\
\hline 26 & & 10 & 87.5 & 0.545 & 7.8 & 0.001908 & 14.31 & \\
\hline 27 & & 10 & 87.5 & 0.545 & 8.4 & 0.001666 & 12.495 & \\
\hline 28 & & 10 & 87.5 & 0.645 & 7.2 & 0.00324 & 24.33 & \\
\hline 29 & & 10 & 87.5 & 0.645 & 7.8 & 0.002623 & 19.6725 & \\
\hline
\end{tabular}

(a) Database Excel Sheet

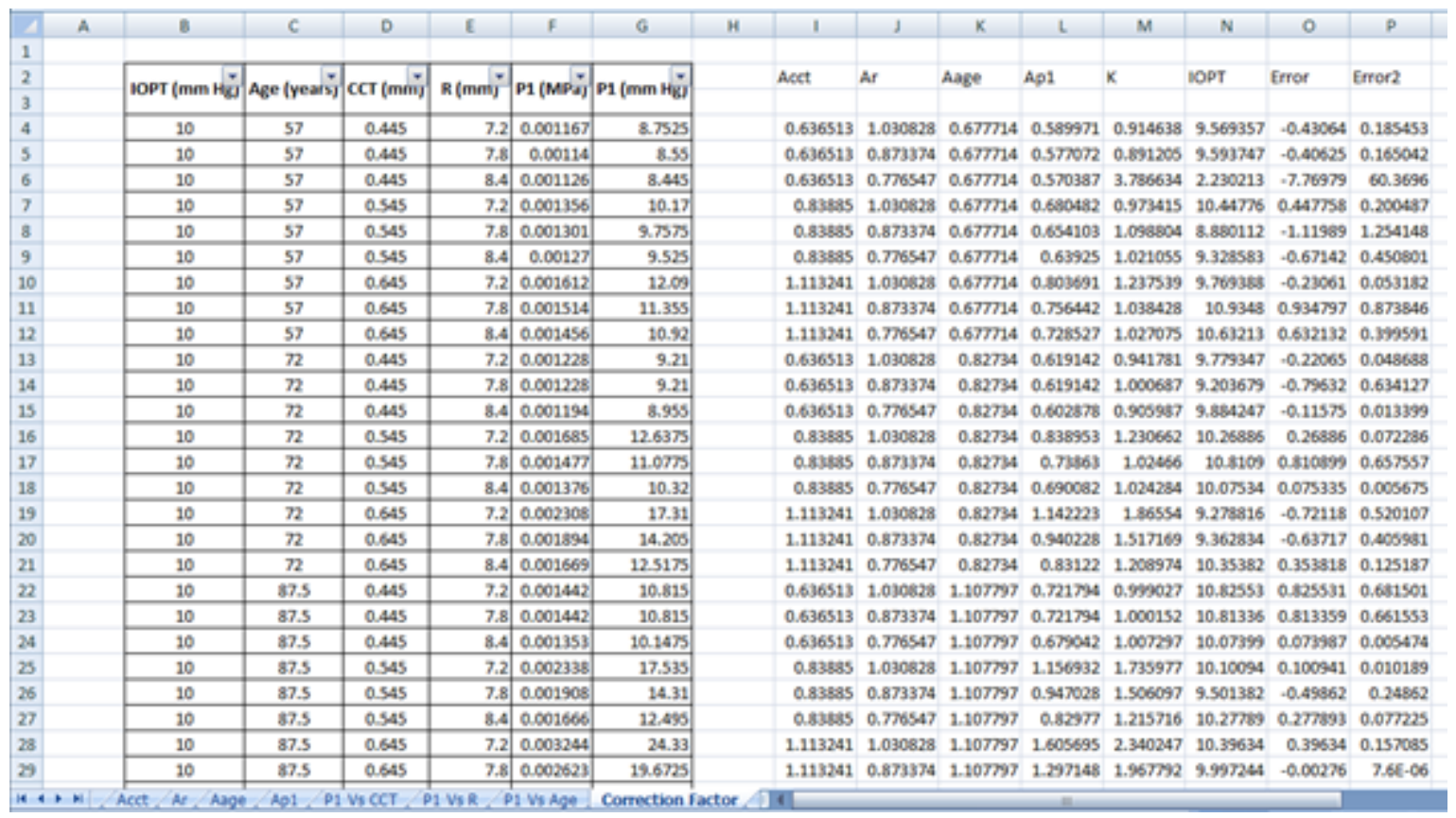

(b) Optimisation process

Fig. 2: Use of spread sheets to develop correction equations. 


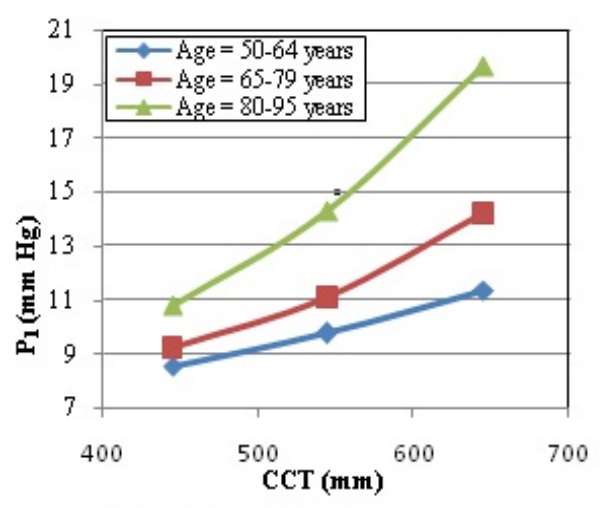

(a) $\mathrm{IOPT}=10 \mathrm{~mm} \mathrm{Hg} 22$

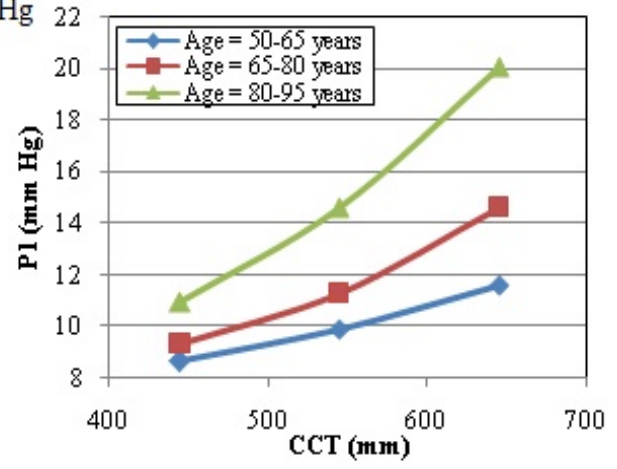

(c) IOPT $=20 \mathrm{~mm} \mathrm{Hg}$

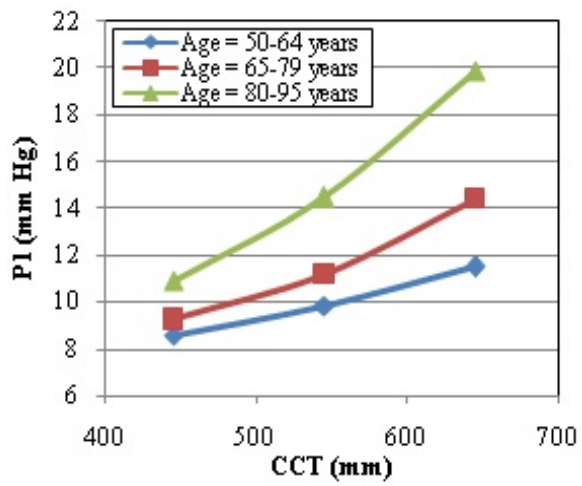

(b) $\mathrm{IOPT}=15 \mathrm{~mm} \mathrm{Hg}$

Fig. 3: Numerical estimation of the effect of CCT on $\mathrm{P}_{1}$ measurement.

The average effect of variation on $\mathrm{P}_{1}$ has been found to be $0.0145 \mathrm{~mm} \mathrm{Hg}, 0.026 \mathrm{~mm} \mathrm{Hg}$ and $0.0449 \mathrm{~mm} \mathrm{Hg}$ per $1 \mu \mathrm{m}$ variation in CCT for corneas with young, middle and old age ranges respectively.

\subsection{Effect of variation in $R$}

The numerical estimation of the effect of corneal curvature $(\mathrm{R})$ on $\mathrm{P}_{1}$ measurements is shown in Fig. 4 for corneas with $\mathrm{R}$ between $7.2 \mathrm{~mm}$ and $8.4 \mathrm{~mm}$; age ranges of young, middle and old (average age = 57, 72 and 87.5 respectively); and IOPT values of $10 \mathrm{~mm} \mathrm{Hg}, 15 \mathrm{~mm} \mathrm{Hg}$ and $20 \mathrm{~mm} \mathrm{Hg}$. The value of CCT has been kept constant at a value of $545 \mu \mathrm{m}$ in all cases.

From Fig. 4 a consistent decrease in $\mathrm{P}_{1}$ measurements with increasing $\mathrm{R}$ can be observed. This could be attributed to the fact that corneal stiffness decreases as the cornea gets flatter.

The average effect of $\mathrm{R}$ variation on $\mathrm{P} 1 \mathrm{has}$ been found to be $0.5451 \mathrm{~mm} \mathrm{Hg}, 1.9833 \mathrm{~mm} \mathrm{Hg}$ and $4.2229 \mathrm{~mm} \mathrm{Hg}$ per 1 $\mathrm{mm}$ change in $\mathrm{R}$ for the young, middle and old age ranges respectively.

\subsection{Effect of variation in age}

Fig. 5 shows the numerical estimation of the effect of age on $\mathrm{P}_{1}$ measurements by considering IOPs of $10 \mathrm{~mm} \mathrm{Hg}, 15 \mathrm{~mm}$ $\mathrm{Hg}$ and $20 \mathrm{~mm} \mathrm{Hg}$. Each set of data includes three lines that correspond to CCT values of $445 \mu \mathrm{m}, 545 \mu \mathrm{m}$ and $645 \mu \mathrm{m}$. 


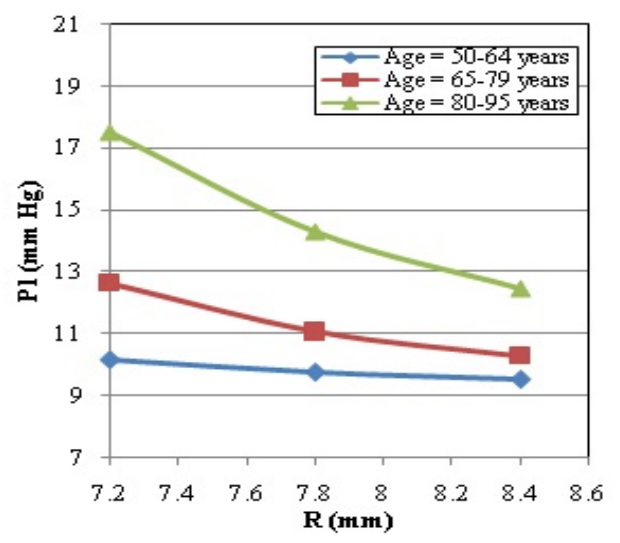

(a) $\mathrm{IOPT}=10 \mathrm{~mm} \mathrm{Hg}$

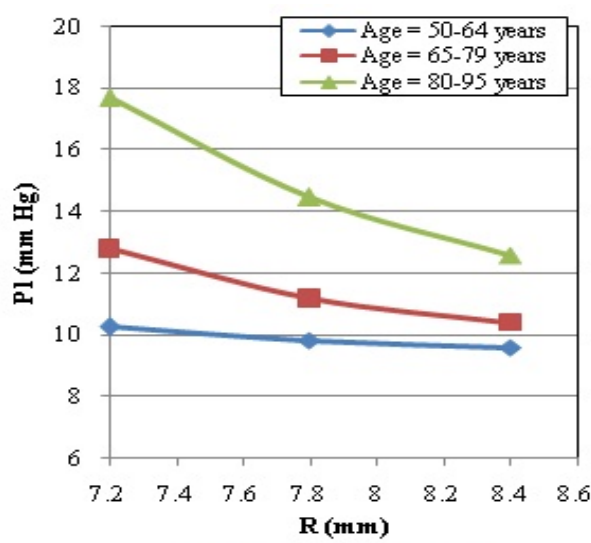

(b) IOPT $=15 \mathrm{~mm} \mathrm{Hg}$

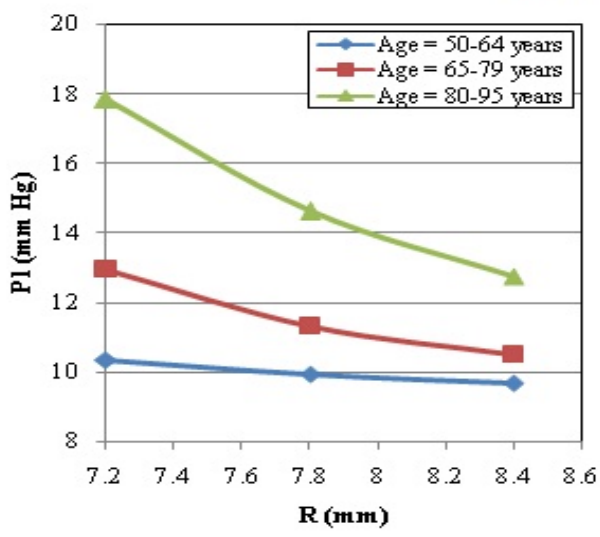

(c) IOPT $=20 \mathrm{~mm} \mathrm{Hg}$

Fig. 4: Numerical estimation of the effect of $\mathrm{R}$ on $\mathrm{P}_{1}$ measurement.

The average increase in $\mathrm{P}_{1}$ values has been found to be $0.7541 \mathrm{~mm} \mathrm{Hg}, 1.515 \mathrm{~mm} \mathrm{Hg}$ and $2.753 \mathrm{~mm} \mathrm{Hg}$ per decade of age for corneas with CCT values of $445 \mu \mathrm{m}, 545 \mu \mathrm{m}$ and $645 \mu \mathrm{m}$ respectively.

\section{Conclusion}

From Fig. 3 it can be seen that for a given CCT, increase in age leads to increase in $\mathrm{P}_{1}$ readings.

Moreover, the effect of $\mathrm{R}$ has been found to become more pronounced with the increase in age and could be attributed to higher material stiffness developed by cornea with increase in age.

Fig. 5 shows a nonlinear increase in $\mathrm{P}_{1}$ measurements with increasing age and could be attributed to the age related material stiffness in cornea.

Acknowledgement: The present work is related to the author's participation in research project under Prof. A Elsheikh at University of Dundee, Scotland, UK; where M Amir also produced his master's dissertation within the current topic. The author would like to express his gratitude towards Prof. A Elsheikh for his supervision and excess to required facilities at Division of Civil Engineering, University of Dundee, Dundee, DD1 4HN, Scotland, UK. 


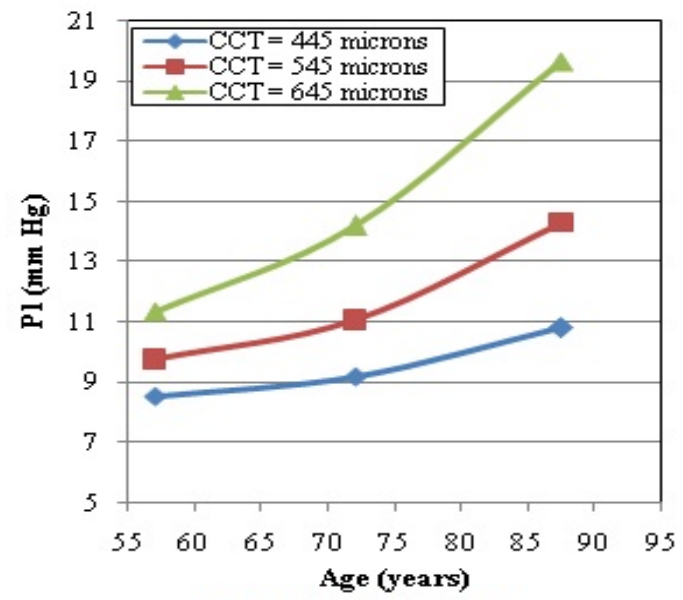

(a) $\mathrm{IOPT}=10 \mathrm{~mm} \mathrm{Hg}$

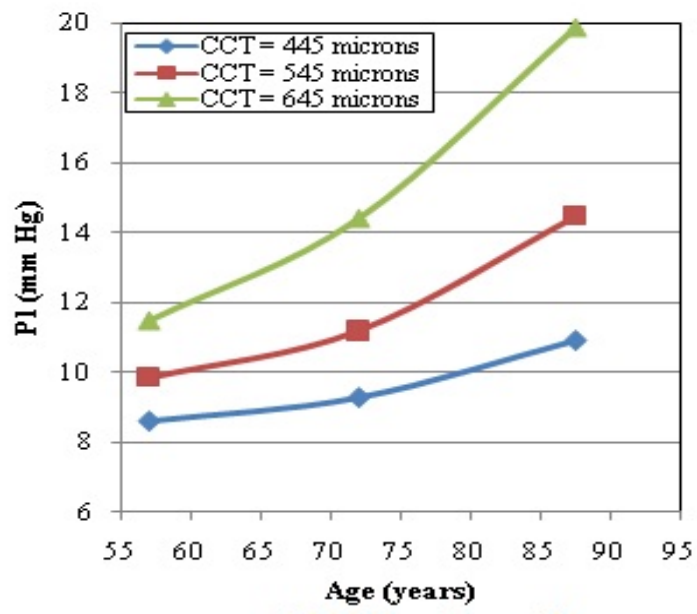

(b) $\mathrm{IOPT}=15 \mathrm{~mm} \mathrm{Hg}$

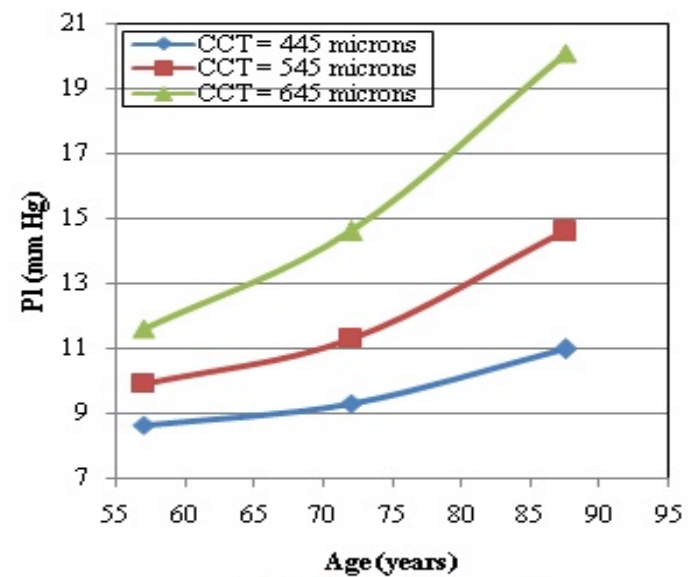

(c) IOPT $=20 \mathrm{~mm} \mathrm{Hg}$

Fig. 5: Numerical estimation of the effect of age on $P_{1}$ measurement.

\section{References}

[1] M.A. Khan, Numerical study on human cornea and modified multiparametric correction equation for Goldmann applanation tonometer, J. Mech. Behav. Biomed. Mater. 30 (2014): 91-102.

[2] J. Morisson, (2003) Glaucoma - A clinical guide.

[3] P.A. Tonnu, T. Ho, K. Sharma, E. White, C. Bunce, and D. Garway-Heath, A Comparison of four methods of Tonometry: Method Agreement and Introbserver Variability, Br. J. Ophthalmol., 89 (2005), 847-850.

[4] N. Ehlers, T. Bramsen, and S. Sperling, Applanation tonometry and central corneal thickness, Acta Ophthalmologica, 53 (1975), 34-43.

[5] M.M. Whitacre, and R. Stein, Sources of Error with use of Goldmann-type Tonometers, Survey of Ophthalmology, 38(1) (1993), 1-30.

[6] J. Liu, and C.J. Roberts, Influence of Corneal Biomechanical Properties on Intraocular Pressure Measurement, J. Cataract Refract. Surg., 31 (2005), 146-155.

[7] J.M. Martinez-de-la-Casa, J. Garcia-Feijoo, A. Fernandez-Vidal, C. Mendez-Hernandez, and J. Garcia-Sanchez, Ocular Response Analyzer versus Goldmann Applanation Tonometry for Intraocular Pressure Measurements, Ophthalmol. Vis. Sci., 47(10) (2006), 4410-4414. 
[8] A. Kotecha, A. Elshiekh, C.R. Roberts, H. Zhu, and D.F. Garway-Heath, Corneal Thickness and Age Related Biomechanical Properties of the Cornea Measured with the Ocular Response Analyzer, Invest. Ophthalmol. Vis. Sci., 47(12) (2006), $5337-5347$.

[9] A. Lam, D. Chen, R. Chiu, and W.S. Chui, Comparison of IOP Measurements between ORA and GAT in Normal Chinese, Optometry and Vision Science, 84(9) (2007), 909-914. 